\title{
ON AN INTEGRAL FORMULA FOR CLOSED HYPERSURFACES OF THE SPHERE
}

\author{
CHORNG-SHI HOUH
}

\begin{abstract}
In a compact oriented hypersurface $M^{n}$ of the sphere $S^{n+1}$ the integral formula $\int_{M^{n}} \nabla K_{r} d V=n \int_{M^{n}}\left(K_{r} K_{1}-K_{r+1}\right) e d V$ is proved where $K_{r}$ is the $r$ th mean curvature, $e$ is the unit normal of $M^{n}$ in $S^{n+1}$. Some applications are considered.
\end{abstract}

1. Let $S^{n+1}$ be the unit sphere in a euclidean space $E^{n+2}$ and $x: M^{n} \rightarrow$ $S^{n+1}$ be an isometric immersion of a compact oriented Riemannian manifold $M^{n}$ of dimension $n$ into $S^{n+1}$. Let $F\left(M^{n}\right), F\left(S^{n+1}\right)$ and $F E\left({ }^{n+2}\right)$ be the bundles of orthonormal frames of $M^{n}, S^{n+1}$ and $E^{n+2}$ respectively. Let $B$ be the set of elements $b=\left(p, e_{1}, \cdots, e_{n}, e, x(p)\right)$ such that $\left(p, e_{1}, \cdots, e_{n}\right) \in$ $F\left(M^{n}\right), \quad\left(x(p), e_{1}, \cdots, e_{n}, e\right) \in F\left(S^{n+1}\right) \quad$ and $\quad\left(x(p), e_{1}, \cdots, e_{n}, e, x\right) \in$ $F\left(E^{n+2}\right)$ with coherent orientations. $d x\left(e_{i}\right)$ is identified with $e_{i}, i=1$, $2, \cdots, n$. Define $\tilde{x}: B \rightarrow F\left(E^{n+2}\right)$ by $\tilde{x}(b)=\left(x(p), e_{1}, \cdots, e_{n}, e, x\right)$.

By the structure equations of $E^{n+2}$ and the pullback by $\tilde{x}$ we may write

$$
d x=\sum \omega_{i} e_{i}, \quad d e=\sum_{i} \theta_{i} e_{i}
$$

with $\theta_{i}=k_{i} w_{i}$. Where $i=1,2, \cdots, n ; k_{1}, \cdots, k_{n}$ are principal curvatures of $M^{n}$ in $S^{n+1}$ at $p$. de does not have component in the $x$ direction is easily followed from $d(e \cdot x)=0$.

2. Let $|\cdot, \cdots, \cdot|$ denote the combined operation of the vector product and exterior product ([1], [3], [4]). Put

$$
\Delta_{r}=|d e \underset{(r \text { times })}{,} \underset{(n-r-1 \text { times })}{d x}, e, x| .
$$

Then

$$
\begin{aligned}
& (-1)^{n-1} d \Delta_{r}=\underset{(r+1 \text { times })}{\mid d e} \underset{(n-r-1 \text { times })}{d x}, \cdots, d x \mid \\
& -|d e \underset{(r \text { times })}{\cdots,} \underset{(n-r \text { times })}{d x}, e|
\end{aligned}
$$

Received by the editors October 21, 1971.

AMS 1970 subject classifications. Primary 53C45, 53B25; Secondary 53A05.

Key words and phrases. Combined operation of the vector product, combined operation of the exterior product, principal curvatures, $r$ th mean curvature, Stokes theorem.

(c.) American Mathematical Society 1972 
Using (1.1) and straight computation we have

and

$$
\underset{(r+1 \text { times })}{\mid d e} \underset{(n-r-1 \text { times })}{d}, \cdots, d x \mid=-n ! K_{r+1} e d V
$$

$$
\mid d e \underset{(r \text { times })}{\cdots} \underset{(n-r \text { times })}{\cdots}, d x, d x, d x ! K_{r} x d V
$$

where $d V=\omega_{1} \wedge \cdots \wedge \omega_{n}$ is the volume element in $M^{n}$ and $K_{r}$ is the $r$ th mean curvature of $M^{n}$ in $S^{n+1}$ defined by the elementary symmetric functions of $k_{1}, \cdots, k_{n}$ as follows:

Thus

$$
\left(\begin{array}{l}
n \\
r
\end{array}\right) K_{r}=\sum_{j_{1}<\cdots<j_{r}} k_{j_{1}} k_{j_{2}} \cdots k_{j_{r}} \quad(1 \leqq r \leqq n) .
$$

$$
d \Delta_{r}=(-1)^{n} n !\left(K_{r+1} e+K_{r} x\right) d V,
$$

and by Stokes' theorem we have

$$
\int_{M^{n}}\left(K_{r+1} e+K_{r} x\right) d V=0, \quad r=1,2, \cdots, n-1 .
$$

This integral formula (2.3) has been obtained by Reilly [5].

3. Substituting (1.1) in the right side of (2.1) yields [1]

$$
\Delta_{r}=(-1)^{n+1} r !(n-r-1) ! \sum_{i=0}^{r}(-1)^{i}\left(\begin{array}{c}
n \\
r-i
\end{array}\right) K_{r-i}{ }^{*} U_{i}
$$

where ${ }^{*}$ is the Hodge star operation and

$$
\begin{gathered}
U_{i} \stackrel{\text { def }}{=} \sum_{j}\left(k_{j}\right)^{i} \omega_{j} e_{j}, \\
{ }^{*} U_{i}=\sum_{j}(-1)^{j-1}\left(k_{j}\right)^{i} \omega_{1} \wedge \cdots \wedge \hat{\omega}_{j} \wedge \cdots \wedge \omega_{n} e_{j},
\end{gathered}
$$

$i=0,1, \cdots, n$. Using (3.1) to calculate $d \Delta_{r}$ we have

$$
\begin{aligned}
d \Delta_{r}=(-1)^{n+1} r !(n-r-1) ! & {\left[\left(\begin{array}{l}
n \\
r
\end{array}\right) d K_{r} \wedge{ }^{*} d x+\left(\begin{array}{l}
n \\
r
\end{array}\right) K_{r} d\left({ }^{*} d x\right)\right.} \\
& \left.+\sum_{i=1}^{r}(-1)^{i}\left(\begin{array}{c}
n \\
r-i
\end{array}\right) d\left(K_{r-i}^{*} U_{i}\right)\right]
\end{aligned}
$$

It is easy to show that

$$
\begin{aligned}
d K_{r} \wedge \wedge^{*} d x & =\nabla K_{r} d V \\
d\left(^{*} d x\right) & =-n\left(K_{1} e+x\right) d V
\end{aligned}
$$


Hence we have

$$
\begin{aligned}
d \Delta_{r}= & (-1)^{n+1} r !(n-r-1) ! \\
& \cdot\left[\left(\begin{array}{c}
n \\
r
\end{array}\right) \nabla K_{r} d V-n\left(\begin{array}{l}
n \\
r
\end{array}\right) K_{1} K_{r} e d V\right. \\
& \left.-n\left(\begin{array}{l}
n \\
r
\end{array}\right) K_{r} x d V+\sum_{i=1}^{r}(-1)^{i}\left(\begin{array}{c}
n \\
r-i
\end{array}\right) d\left(K_{r-i}^{*} U_{i}\right)\right] .
\end{aligned}
$$

On the other hand by (3.1) and (2.2) we obtain

$$
\begin{aligned}
& d x \cdot \Delta_{r}=(-1)^{n+1} r !(n-r-1) ! \sum_{i=0}^{r}\left[(-1)^{i}\left(\begin{array}{c}
n \\
r-i
\end{array}\right) K_{r-i} \sum_{j}\left(k_{j}\right)^{i}\right] d V, \\
& x \cdot d \Delta_{r}=(-1)^{n} n ! K_{r} d V .
\end{aligned}
$$

From $x \cdot \Delta_{r}=0$ it follows $0=d\left(x \cdot \Delta_{r}\right)=d x \cdot \Delta_{r}+x d \Delta_{r}$ and hence

$$
r\left(\begin{array}{l}
n \\
r
\end{array}\right) K_{r}-\sum_{i=1}^{r}(-1)^{i}\left(\begin{array}{c}
n \\
r-i
\end{array}\right) K_{r-i} \sum_{j}\left(k_{j}\right)^{i}=0 .
$$

This is the well-known identity of Newton.

Since $d\left(K_{r}^{*} d x\right)=\nabla K_{r} d V-n K_{r}\left(K_{1} e+x\right) d V$, one obtains by the Stokes' theorem

$$
\int_{M^{n}}\left[\nabla K_{r}-n K_{r}\left(K_{1} e+x\right)\right] d V=0 .
$$

Together with (2.3) we have the following theorem.

THEOREM. Let $M^{n}$ be a compact oriented hypersurface in $S^{n+1}, K_{r}$ be the rth mean curvature of $M^{n}$ in $S^{n+1}$, e be the unit normal of $M^{n}$ in $S^{n+1}$. Then

$$
\int_{M^{n}} \Gamma K_{r} d V^{\prime}=n \int_{M l^{n}}\left(K_{r} K_{1}-K_{r+1}\right) e d V .
$$

4. The following are some applications of the theorem.

COROLLARY 1. In the theorem suppose, furthermore, that there is a fixed vector $a$ in $E^{n+2}$ such that the function a $e$ is of the same sign on $M^{n}, K_{i}>0$ for $i=1, \cdots, r, 1 \leqq r \leqq n-1$, and $K_{r}$ is constant. Then $M^{n}$ is a hypersphere in $S^{n+1}$.

Proof. Under the assumption we have that $K_{r} K_{1}-K_{r+1}=0$. The same argument as in [4, p. 731] yields $k_{1}=k_{2}=\cdots=k_{n}$ at all points of $M^{n}$. Hence $M^{n}$ is a hypersphere in $S^{n+1}$. 
COROLlARY 2. In the theorem suppose, furthermore, that $M^{n}$ is minimal in $S^{n+1}$ and that there is a fixed vector $a$ in $E^{n+2}$ such that the function $a \cdot e$ is of the same sign on $M^{n}$. Then $M^{n}$ is totally geodesic.

Proof. By the assumptions and (3.4) it implies that $K_{1}=K_{2}=0$. So $k_{i}=0(i=1,2, \cdots, n)$ and $M^{n}$ is totally geodesic. This result is known $[2$, p. 33].

The author would like to express his thanks to Dr. B. Y. Chen for the valuable conversations.

\section{REFERENCES}

1. B. Y. Chen, Some integral formulas for hypersurfaces in Euclidean space, Nagoya Math. J. 43 (1971), 117-125.

2. S. S. Chern, Minimal submanifolds in a Riemannian manifold, University of Kansas, Department of Mathematics Technical Report, 19, Univ. of Kansas, Lawrence, Kan., 1968. MR 40 \#1899.

3. C. C. Hsiung, Some integral formulas for closed hypersurfaces, Math. Scand. 2 (1954), 286-294. MR 16, 849.

4. C. C. Hsiung and J. K. Shahin, Affine differential geometry of closed hypersurfaces, Proc. London Math. Soc. (3) 17 (1967), 715-735. MR 36 \#2069.

5. R. C. Reilly, Extrinsic rigidity theorems for compact submanifolds of the sphere, J. Differential Geometry 4 (1970), 487-497.

Department of Mathematics, Wayne State University, Detroit, Michigan 48202 